Recommandations pour la pratique clinique

\title{
Prise en charge initiale du cancer épithélial de l'ovaire. Introduction aux Recommandations de pratiques cliniques françaises communes de FRANCOGYN, CNGOF, SFOG, GINECO-ARCAGY et labélisées par l'INCa
}

\author{
Management of Epithelial Ovarian Cancer : French joint recommendations of \\ FRANCOGYN, CNGOF, SFOG, GINECO-ARCAGY and endorsed by INCa. Introduction
}

\author{
V. Lavoué ${ }^{\mathrm{a}, \mathrm{b}}$, C. Huchon ${ }^{\mathrm{c}}, \mathrm{E}$. Daraï ${ }^{\mathrm{d}, *, \mathrm{e}}$ \\ a Service de gynécologie, CHU de Rennes, hôpital Sud, 16, boulevard de Bulgarie, 35000 Rennes, France \\ ${ }^{\mathrm{b}}$ Inserm 1242, oncogenesis, stress and signaling, CRLC Eugène Marquis, université de Rennes 1, 35000 Rennes, France \\ ' Service de gynécologie et obstétrique, CHI de Poissy-St-Germain-en-Laye, université Versailles-Saint-Quentin en Yvelines, 10, rue du champ Gaillard, \\ BP 3082, 78303 Poissy cedex, France \\ ${ }^{\mathrm{d}}$ Service de gynécologie-obstétrique et médecine de la reproduction, hôpital Tenon, Assistance publique des hôpitaux de Paris (AP-HP), 4, rue de la Chine, \\ 75020 Paris, France \\ e UMRS-938, faculté de médecine UPMC, centre cancer associé à la grossesse (CALG), institut universitaire de cancérologie (IUC), Sorbonne université, \\ 75000 Paris, France
}

\section{R É S U M É}

Le cancer de l'ovaire, de la trompe et du péritoine primitif a une prise en charge hétérogène en fonction de la structure de prise en charge ou encore en fonction des patientes, par exemple entre les patientes âgées et les personnes plus jeunes. Ces différentes modalités de prise en charge donnent un pronostic hétérogène pour les patientes atteintes d'un cancer de l'ovaire. La proposition de recommandations nationales basées sur les preuves est nécessaire pour essayer d'homogénéiser les pratiques pour rendre un meilleur pronostic aux patientes.

\section{A B S T R A C T}

Ovarian cancer had a poor prognosis that could be heterogeneous according specialized center or not and according elderly in comparison with their younger counterpart. National recommendations are required to propose homogeneous practice and increase overall ovarian cancer prognosis.

Le cancer épithélial de l'ovaire, des trompes et du péritoine primitif affecte une femme sur 70 dans les pays industrialisés. Son incidence est d'environ 4700 nouveaux cas en France en 2015. Il est le $5^{\mathrm{e}}$ cancer le plus fréquent chez la femme et représente la $4^{\mathrm{e}}$ cause de mortalité par cancer chez la femme, avec 3200 décès par an. Il concerne majoritairement les femmes après la ménopause. Le pronostic du cancer de l'ovaire reste sombre avec une survie globale à 5 ans de $30 \%$, tout stades confondus et la majorité des décès intervient dans les deux premières années après le

\footnotetext{
* Auteur correspondant.

Adresse e-mail : emile.darai@aphp.fr (E. Daraï).
}

diagnostic. Les trois quarts des patientes sont diagnostiquées à un stade avancé (stade IIIc et IV de la Fédération internationale de gynécologie obstétrique - FIGO), c'est-à-dire, avec une diffusion de la maladie au-delà des ovaires, sur toute la surface du péritoine. Ces patientes au stade IIIc-IV de la maladie ont une survie globale à 5 ans inférieure à $20 \%$.

La prise en charge des cancers de l'ovaire, de la trompe et du péritoine primitif, surtout à un stade avancé, est hétérogène en France tant sur le plan du traitement chirurgical que sur le plan du traitement chimiothérapeutique ou de la séquence thérapeutique (chirurgie première versus chirurgie d'intervalle). L'effort chirurgical réalisé conditionne à la fois le pronostic oncologique de la 
patiente par sa capacité à rendre ou non un résidu tumoral nul et les morbi-mortalités périopératoires. Cette modulation du bénéfice de la chirurgie de cytoréduction est corrélée à la structure de prise en charge avec notamment le nombre de chirurgie pour stade avancé réalisée. Plus le volume est important (seuil à 20 chirurgies par an sur 2 chirurgiens) et plus le taux de chirurgie complète obtenu augmente et le taux de complications périopératoires diminue. Cependant, malgré la mise en place de seuil de chirurgie oncologique pelvienne (20 procédures oncologiques par an), on observe encore une grande dispersion de la prise en charge chirurgicale des patientes atteintes de cancer de l'ovaire. Par ailleurs, le traitement par chimiothérapie peut être effectué par différente voie d'abord, avec un bénéfice positif montré pour l'approche intrapéritonéale soit sur une modalité de chimiothérapie intrapéritonéale soit sur une chimiothérapie hyperthermique intrapéritonéale. Dans les deux cas, l'approche intrapéritonéale a objectivé des bénéfices en termes de survie globale, mais est finalement utilisée de manière très inégale, voire parcimonieuse, sur le territoire français. De même, l'utilisation de thérapie ciblée comme le bévacizumab est variablement utilisé. Il s'agit d'un traitement anti-angiogénique prescrit en entretien sur 15 mois et qui a montré un bénéfice en survie sans récidive, surtout pour les patientes de plus mauvais pronostic (C'est-à-dire, avec résidu tumoral post chirurgie, patiente non opérée ou encore avec un stade FIGO IV). Son utilisation peut être utilisée massivement dans certains centres avec une certaine toxicité (20\% de complications de grade III, principalement des complications à type d'hypertension artérielle) ou simplement réservé au cas de plus mauvais pronostic dans d'autres centres.

Malgré l'établissement de ces critères de qualités structuraux, de critères de processus, et de critère de résultats, la situation n'a pas évolué en France au détriment des patientes. Le but de cette recommandation pour la pratique clinique (RPC) est de permettre une prise en charge optimale des patientes ayant un cancer épithélial de l'ovaire, de la trompe ou du péritoine primitif, dont l'objectif ultime est l'amélioration de la survie et de la qualité de vie des patientes tout en optimisant le coût en santé publique. Ces RPC ont été conduite par un groupe de travail réuni par le groupe français de recherche en chirurgie oncologique et gynécologique (FRANCOGYN), le collège national des gynécologues obstétriciens français (CNGOF), la Société française d'oncologie gynécologique (SFOG) et le groupe d'investigateurs national des études des cancers ovariens et du sein (GINECO-ARCAGY) pour la prise en charge initiale des cancers épithéliaux de l'ovaire, tubaires ou péritonéaux primitifs (à l'exclusion de la récidive du cancer de l'ovaire ou des tumeurs borderlines). L'élaboration de ces RPC répond aux normes fixées par la Haute Autorité de santé (HAS) et de l'institut national du cancer (INCa) avec des relectures internes et externes au groupe de travail.

\section{Déclaration de liens d'intérêts}

Les auteurs déclarent ne pas avoir de liens d'intérêts. 